\title{
Efeito da crioterapia na resposta eletromiográfica dos músculos tibial anterior, fibular longo e gastrocnemio lateral de atletas após o movimento de inversão do tornozelo
}

Effect of cryotherapy on the electromyographic response of the tibialis anterior, peroneus longus and gastrocnemius lateralis muscles in athletes after ankle inversion movement

Efecto de la crioterapia en la respuesta electromiográfica de los músculos tibial anterior, fibular largo y gastrocnemio lateral de atletas después del movimiento de inversión del tobillo Carolina Saenz Alonso', Christiane de Souza Guerino Macedo', Rinaldo Roberto de Jesus Guirro,2,

RESUMO I A crioterapia é utilizada para tratamento de traumas no tornozelo/pé em atletas; entretanto, sua ação sobre o controle motor apresenta controvérsias. Este estudo objetivou verificar o efeito da crioterapia na resposta eletromiográfica dos músculos do tornozelo de atletas após inversão. A amostra foi composta por 20 voluntários: 10 universitários atletas de basquetebol e 10 universitários não atletas. Todos foram submetidos à inversão do tornozelo em plataforma para a análise do sinal eletromiográfico dos músculos Gastrocnêmio Lateral (GL), Tibial Anterior (TA) e Fibular Longo (FL), pré e pós-crioterapia por imersão do tornozelo, a $4 \pm 2^{\circ} \mathrm{C}$, por 20 minutos. A análise estatística utilizou o teste de Shapiro Wilk, Wilcoxon e Mann-Whitney, com nível de significância em 5\%. Considerou-se o pico de RMS, o RMS após 0,2 segundos e após 1,0 segundo da abertura da plataforma de inversão. Todos os dados foram normalizados pelo pico do RMS no momento précrioterapia. Evidenciou-se diminuição do pico do RMS e do RMS até 0,2 s para o TA nos atletas e nos músculos GL e FL dos não atletas. Após um segundo da abertura da plataforma houve menor recrutamento dos músculos GL, TA e FL para os não atletas. A comparação entre atletas e não atletas apontou, no RMS até 1 segundo, menor recrutamento para o GL dos atletas pré-crioterapia e TA pré e após o resfriamento. Conclui-se que a crioterapia diminuiu a resposta eletromiográfica do músculo TA de atletas e GL, TA e FL de não atletas.

Descritores I Crioterapia; Eletromiografia; Tornozelo; Atletas; Fisioterapia.
ABSTRACTICryotherapy is often indicated in the treatment of ankle/foot injuries in athletes; however, there is some controversies regarding its action on motor control. The main objective was to evaluate the effects of cryotherapy on electromyographic response of the ankle muscles in athletes after inversion. The sample consisted of 20 volunteers: 10 university basketball athletes and 10 non-athletes. All athletes were submitted to ankle inversion for the analysis of the EMG signals of Lateral Gastrocnemius (LG), Tibilis Anterior (TA) and Fibular Longus (FL) muscles, before and immediately after ankle immersion in cold water at $4 \pm 2^{\circ} \mathrm{C}$, for 20 minutes. The peak Root Mean Square (RMS) values were analyzed between $\mathrm{O}$ to 0.2 seconds and between $\mathrm{O}$ to $1 \mathrm{sec}-$ ond after a sudden inversion on a tilting platform. Data were normalized by the peak RMS measured before cold water immersion. The statistical analysis was performed using Shapiro Wilk, Wilcoxon and Mann-Whitney tests. The level of significance was set at $5 \%$. The results showed that the RMS values were lower in TA muscle of athletes, and up to 0.2 seconds in $L G$ and FL muscles of non-athletes; up to 1 second, there was lower LG, TA and FL muscle recruitment in non-athletes. The comparison between athletes and nonathletes showed, in RMS up to 1 second, lower GL muscle recruitment in athletes submitted to pre cold water immersion and TA muscle pre and after cooling. Cryotherapy decreased the electromyographic response of TA muscle in athletes and of LG, TA and FL muscles in non-athletes.

Keywords I Cryotherapy; Eletromyography; Ankle; Athletes; Physical Therapy Specialty.

Estudo desenvolvido no Centro de Reabilitação do Hospital das Clínicas da Faculdade de Medicina de Ribeirão Preto da Universidade de São Paulo (FMRP/USP) - Ribeirão Preto (SP), Brasil.

'Laboratório de Recursos Fisioterapêuticos da FMRP/USP - Ribeirão Preto (SP), Brasil.

${ }^{2}$ Departamento de Biomecânica, Medicina e Reabilitação do Aparelho Locomotor da FMRP/USP - Ribeirão Preto (SP), Brasil.

\footnotetext{
Endereço para correspondência: Rinaldo Roberto de Jesus Guirro - Universidade de São Paulo - Faculdade de Medicina de Ribeirão Preto - Avenida Bandeirantes, 3.900 CEP: 14049-900 - Ribeirão Preto (SP), Brasil - E-mail: rguirro@fmrp.usp.br

Apresentação: fev. 2013 - Aceito para publicação: out. 2013 - Fonte de financiamento: CNPq e FAPESP - Conflito de interesses: nada a declarar - Parecer de aprovação no Comitê de Ética no 2968/2010 (FMRP/USP); NCT01870414
} 
RESUMEN I La crioterapia es utilizada para tratamiento de traumas en el tobillo/pie en atletas; entre tanto, su acción sobre el control motor presenta controversias. Este estudio intentó verificar el efecto de la crioterapia en la respuesta electromiográfica de los músculos del tobillo de atletas después de inversión. La muestra fue compuesta por 20 voluntarios: 10 universitarios atletas de básquetbol y 10 universitarios no atletas. Todos fueron sometidos a inversión del tobillo en plataforma para el análisis de la señal electromiográfica de los músculos Gastrocnemio Lateral (GL), Tibial Anterior (TA) y Fibular Largo (FL), pre y post-crioterapia por inmersión del tobillo, a $4 \pm 2^{\circ} \mathrm{C}$, durante 20 minutos. El análisis estadístico utilizó el test de Shapiro Wilk, Wilcoxon y Mann-Whitney, con nivel de significación de 5\% Se consideró el pico de RMS, el RMS después de 0,2 segundos y después de 1,0 segundo de la apertura de la plataforma de inversión. Todos los datos fueron normalizados por el pico del RMS en el momento pre-crioterapia. Se evidenció disminución del pico del RMS y del RMS hasta O,2 s para el TA en los atletas y en los músculos GL y FL de los no atletas. Después de un segundo de la apertura de la plataforma hubo menor reclutamiento de los músculos GL, TA y FL para los no atletas. La comparación entre atletas y no atletas señaló, en el RMS hasta 1 segundo, menor reclutamiento para el GL de los atletas pre-crioterapia y TA pre y después del enfriamiento. Se concluye que la crioterapia disminuyó la respuesta electromiográfica del músculo TA de atletas y GL, TA y FL de no atletas.

Palabras clave I Crioterapia; Eletromiografía; Tobillo; Atletas; Fisioterapia.

\section{INTRODUÇÃO}

A articulação do tornozelo representa $34,3 \%$ de todas as lesões relacionadas ao esporte ${ }^{1}$, onde a entorse em inversão é também a mais comum entre indivíduos fisicamente ativos ${ }^{2}$. Estudos epidemiológicos estimam que a taxa de incidência de entorses de tornozelos na população em geral é cerca de 5 a 7 torções a cada 1.000 pessoas por ano; mundialmente, aproximadamente uma entorse de tornozelo ocorre por dia a cada 10.000 pessoas $^{3}$.

Após a lesão no tornozelo a crioterapia é indicada como tratamento para atletas de elite e amadores, com objetivos terapêuticos específicos ${ }^{4}$. Afirma-se sua eficácia na diminuição da dor, edema local, inflamação, fluxo sanguíneo, taxa metabólica, temperatura intramuscular, hipertonicidade e velocidade da condução nervosa ${ }^{5}$. Várias são as formas de utilização, como imersão em água gelada, resfriamento termoelétrico, gelo seco e pacotes de gelo, com tempos de aplicação estimados entre 15 e 30 minutos $^{6}$.

Sabe-se que o uso terapêutico do frio reduz gradativamente a transmissão de impulsos nos nervos sensitivos, em função da redução na velocidade de condução das fibras nervosas ${ }^{7}$, o que diminui a propriocepção e aumenta a predisposição do indivíduo a lesões devido à menor produção de força muscular, aferências proprioceptivas ou uma combinação destes fatores ${ }^{8}$. Os efeitos prejudiciais da crioterapia no controle motor também são apontados por desenvolver a redução do torque muscular, inadequado feedback periférico para a propriocepção e alteração das propriedades biomecânicas das articulações, resultando em lesões, quando o exercício é praticado ${ }^{9}$. Ainda, uma sobrecarga na execução de exercícios após o resfriamento do músculo pode levar a nova lesão muscular, uma vez que o controle motor está alterado ${ }^{10}$.
Para avaliar o efeito da crioterapia sobre o recrutamento muscular pode-se utilizar a eletromiografia de superfície. Nesse sentido, Cordova et al. ${ }^{11}$ e Berg et al. ${ }^{12}$ observaram que o resfriamento não alterou o tempo de latência ou a amplitude da resposta do músculo fibular longo ao movimento de inversão do tornozelo. Por outro lado, Schmid, Moffat e Gutierrez ${ }^{13}$ afirmam que, apesar de controvérsias, alguns estudos apontam que a crioterapia diminui a atividade muscular, com altos índices de lesões no retorno a jogos esportivos. Considerando os dados contraditórios em relação à crioterapia e resposta eletromiográfica, e ainda que na prática esportiva muitos atletas retornem imediatamente às atividades competiti$\operatorname{vas}^{14}$, a hipótese do presente estudo é que o resfriamento do pé e tornozelo diminui a resposta eletromiográfica dos músculos relacionados à articulação durante o movimento de inversão. Dessa forma, o objetivo foi apontar o efeito da crioimersão do pé e tornozelo no pico da resposta eletromiográfica dos músculos GL, TA e FL, após o movimento passivo de inversão do tornozelo de atletas.

\section{METODOLOGIA}

A pesquisa foi realizada no Laboratório de Análise do Movimento do Centro de Reabilitação do Hospital das Clínicas da Faculdade de Medicina de Ribeirão Preto da Universidade de São Paulo (FMRP-USP), em conformidade com a Resolução 466/2012 do Conselho Nacional de Saúde, sendo o projeto aprovado pelo Comitê de Ética em Experimentação com Seres Humanos do Hospital das Clínicas-FMRP/USP (Plataforma Brasil - 2968/2010) e registrado como ensaio clínico (Clinical Trial/NCT01870414). 
Para compor a amostra, de conveniência, foram recrutados 20 sujeitos, gênero masculino, universitários, idade entre 18 a 28 anos, IMC entre 20 e $24 \mathrm{~kg} / \mathrm{m}^{2}$, sem história de lesões agudas dos membros inferiores e sem queixas de dor. Destes, 10 eram atletas universitários de basquetebol, em treinamento esportivo de no mínimo três dias por semana, sem afastamento da prática esportiva nos últimos seis meses, e formaram o grupo atletas. Os outros 10 voluntários eram indivíduos saudáveis, ativos e formaram o grupo não atletas.

Foram excluídos, no momento do recrutamento, indivíduos submetidos a procedimentos cirúrgicos ou que apresentassem lesões musculares ou articulares nos membros inferiores nos últimos seis meses; indivíduos com diagnóstico de patologias metabólicas, reumáticas ou ortopédicas incapacitantes; com desordens de equilibrio ou cognitiva; indivíduos que apresentassem queixas de fadiga muscular no momento dos testes ou necessitassem do uso de estabilizadores para realizá-los; lesões cutâneas de membros inferiores e hipersensibilidade ao frio. Por se tratar de uma amostra de conveniência não houve perda amostral.

A coleta de dados foi desenvolvida com o eletromiógrafo Myomonitor IV (Delsys ${ }^{\circledR}$, Boston, MA, USA), impedância de $10^{9} \mathrm{Ohms}$, resolução de 16 bits, faixa de entrada de $\pm 1 \mathrm{~V}$, frequência de amostragem de $1000 \mathrm{~Hz}$, passa banda de $20-450 \mathrm{~Hz}$, taxa de ruído do sinal $\leq 1.2 \mathrm{uV}$ RMS, interfaciado com microcomputador, com ganho de 1.000 vezes. Os sensores de superfície simples diferencial (Delsys ${ }^{\circledR}$, Boston, MA, USA) apresentavam barras de contato de prata $(10 \times 1 \mathrm{~mm})$, espaçadas em $10 \mathrm{~mm}$, ganho amplificado em $10 \mathrm{~V} / \mathrm{V} \pm 1 \%$, Índice de Rejeição por Mudulação Comum de $92 \mathrm{~dB}$ e impedância $>10^{15} \Omega / / 0,2 \mathrm{pF}$. Para a aquisição, armazenamento e análise foi utilizado o Data Acquisition Software (Delsys ${ }^{\circledR}$, Boston, MA, USA).

Para a colocação dos eletrodos, o voluntário permaneceu em pé, onde foi realizada a tricotomia, limpeza da pele e acoplamento dos sensores sobre os ventres dos músculos GL, TA e FL, como proposto pelo Surface EMG for Non-Invasive Assessment of Muscles (SENIAN), fixados por adesivo dupla face e faixa elástica. $\mathrm{Na}$ sequência, o sujeito foi posicionado sobre uma plataforma de inversão, em ortostatismo, olhos abertos e pés descalços (Figura $1 \mathrm{~A}$ ), sincronizada com o eletromiógrafo e ativada pelo computador. A coleta consistiu de seis movimentos repentinos e inesperados de inversão do tornozelo a 30 graus (Figura 1B), três movimentos para cada lado, de forma aleatória. A coleta eletromiográfica foi realizada por avaliador cego que não participou da análise, tabulação e descrição dos dados.
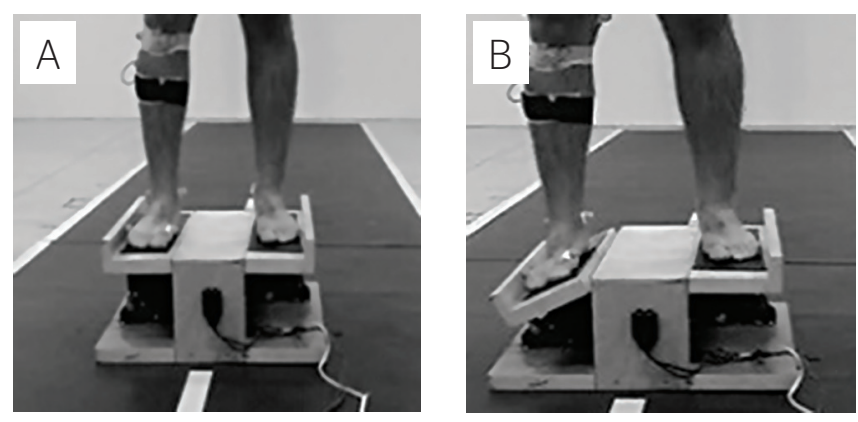

Figura 1. Momento inicial (A) e final (B) da abertura da plataforma de inversão a 30 graus

Após a avaliação eletromiográfica inicial, os sujeitos foram posicionados sentados, com as articulações do quadril, joelho e tornozelo em 90 graus; e submeteram a extremidade do membro inferior dominante à crioterapia por imersão, por 20 minutos, com lâmina de água em 20 centímetros, em temperatura de $4 \pm 2^{\circ} \mathrm{C}$, controlada por termômetro digital infravermelho (MultTemp ${ }^{\circledR}$, Porto Alegre, RS, Brasil). A lâmina de água foi localizada abaixo da fixação dos eletrodos para a coleta eletromiográfica; assim, os mesmos não necessitaram ser retirados. Imediatamente após o término da aplicação da crioterapia foi repetida a coleta eletromiográfica, como descrito anteriormente.

Buscou-se a atividade elétrica, em RMS (Root Mean Square), dos músculos GL, TA e FL do membro inferior dominante, submetidos ao movimento de inversão a 30 graus (pré e pós-aplicação de crioterapia). Todos os dados foram normalizados pelo pico do RMS no momento pré-crioterapia, sendo considerado para a análise o pico do RMS, o RMS após 0,2 segundos e após 1 segundo da abertura da plataforma. A análise estatística foi desenvolvida no software $\operatorname{SPSS}^{\circledR}$ 15.0. A análise da normalidade utilizou o teste de Shapiro Wilk e, para a comparação intra e intergrupos, os testes de Wilcoxon e Mann-Whitney U. O nível de significância foi estabelecido em 5\%.

\section{RESULTADOS}

Os dados antropométricos da amostra estão apresentados na Tabela 1. A comparação entre os dois grupos (atletas e não atletas) não apontou diferenças para as variáveis antropométricas analisadas.

Os resultados dos valores do pico do RMS para os músculos GL, TA e FL de atletas e não atletas após a abertura da plataforma de inversão, normalizados pelo pico pré-crioterapia, estão apresentados na Tabela 2. Para os atletas, a comparação pré e pós-crioterapia apontou 
$p=0,285$ para o GL, $p=0,017$ no TA e $p=0,333$ para o FL. Para o grupo dos não atletas, $p=0,007, p=0,093$ e $\mathrm{p}=0,013$, para os mesmos músculos, respectivamente.

A resposta eletromiográfica (em RMS) coletada até 0,2 segundos após a abertura da plataforma de inversão está demonstrada na Tabela 2. A análise estatística apontou para os músculos GL, TA e FL os valores de $\mathrm{p}=0,24$, $\mathrm{p}=0,007$ e $\mathrm{p}=0,241$, respectivamente, para os atletas; e $\mathrm{p}=0,037, \mathrm{p}=0,114$ e $\mathrm{p}=0,022$ para os não atletas.

Já o RMS coletado até um segundo após a abertura da plataforma de inversão não observou diferenças no grupo atletas para os músculos GL ( $\mathrm{p}=0,241), \mathrm{TA}(\mathrm{p}=0,074)$ e $\mathrm{FL}(\mathrm{p}=0,169)$. Porém, para os não atletas houve diferença para o GL ( $\mathrm{p}=0,007) \mathrm{TA}(\mathrm{p}=0,007)$ e FL ( $\mathrm{p}=0,013)$, após a aplicação da crioimersão (Tabela 2).

Por fim, a comparação entre atletas e não atletas estabeleceu diferença significativa somente para o RMS analisado até 1 segundo após a abertura da plataforma, onde os atletas mostraram menor recrutamento para o GL pré-crioterapia $(\mathrm{p}=0,01)$ e para o musculo TA pré e pós-crioterapia $(\mathrm{p}=0,05)$. Para as demais análises não foram observadas diferenças entre os grupos.

\section{DISCUSSÃO}

Os resultados apontam que após crioimersão ocorreu diminuição da resposta eletromiográfica para atletas

Tabela 1. Média e desvio padrão da idade e dados antropométricos de universitários atletas de basquetebol e não atletas. $n=10$

$\begin{array}{lccc} & \begin{array}{c}\text { Atletas } \\ \text { média } \pm \mathrm{DP}\end{array} & \begin{array}{c}\text { Não atletas } \\ \text { média } \pm \mathrm{DP}\end{array} & \text { Valor } \mathrm{p} \\ \text { Idade (anos) } & 24,1 \pm 6,93 & 21,3 \pm 1,55 & 0,14 \\ \text { Massa }(\mathrm{kg}) & 84,6 \pm 8,24 & 74,86 \pm 13,63 & 0,07 \\ \text { Estatura }(\mathrm{cm}) & 181,44 \pm 8,47 & 175,8 \pm 5,02 & 0,16 \\ \text { IMC }\left(\mathrm{kg} / \mathrm{m}^{2}\right) & 25,94 \pm 2,02 & 23,69 \pm 3,77 & 0,13\end{array}$

DP: desvio padrão. e não atletas, o que confirma a hipótese inicial do estudo. Estes resultados são justificados em função de a crioterapia reduzir a amplitude e aumentar a latência do potencial de ação do nervo ${ }^{15}$, o que desencadeia alterações na estrutura da membrana do axônio e na condutância dos canais de sódio e potássio, com redução da condução nervosa ${ }^{16}$, principalmente dos nervos sensitivos ${ }^{17}$. Estes achados são confirmados por Khanmohammadi et al. ${ }^{18}$, que afirmam existir uma relação linear entre o nível de recrutamento do fuso muscular e a diminuição da temperatura. Também, Bleakley et al. ${ }^{19}$, em uma revisão sistemática, sugerem que após o resfriamento tecidual de 20 minutos os atletas podem apresentar um déficit de desempenho e necessitam de um progressivo aquecimento para o retorno ao esporte.

A relação entre crioterapia e diminuição do controle motor em atletas de basquetebol deve ser melhor estudada e discutida, já que na tentativa de minimizar os danos teciduais das lesões agudas do tornozelo este recurso é frequentemente utilizado no tratamento de lesões em tecidos moles ${ }^{20}$. Sabe-se que o uso de gelo, compressão e elevação tem sido amplamente aceito como tratamento padrão para controle da dor e edema, o que facilita a atividade precoce de sustentação do peso e retorno antecipado ao esporte. Entretanto, o efeito da crioterapia no controle motor não está totalmente esclarecido, acredita-se que pode afetar a velocidade de condução nervosa, a latência do reflexo, e taxa de descarga do fuso muscular, resultando em uma resposta eferente inadequada ${ }^{12}$.

Nossos resultados contrastam com os de Berg et al. ${ }^{12}$ e Cordova et al. ${ }^{11}$, que não observaram diferenças na amplitude ou latência do músculo fibular longo durante o movimento de inversão após o resfriamento da articulação do tornozelo com bolsas de gelo. Porém, para Costello et al. ${ }^{21}$, médicos, fisioterapeutas e preparadores físicos devem ser cautelosos quanto ao retorno de

Tabela 2. Valores do Root Mean Square pré e pós-crioimersão, expressos em porcentagem (mediana; valores mínimos e máximos), normalizados pelo pico do Root Mean Square no momento pré-crioimersão, nos tempos pico, 0,2 s e 1,0 s após a abertura da plataforma de inversão, para os músculos Gastrocnêmio Lateral, Tibial Anterior e Fibular Longo, dos grupos atletas e não atletas. n=10

\begin{tabular}{|c|c|c|c|c|c|c|c|}
\hline & & \multicolumn{3}{|c|}{ Atletas } & \multicolumn{3}{|c|}{ Não atletas } \\
\hline & & \multicolumn{3}{|c|}{ Músculos } & \multicolumn{3}{|c|}{ Músculos } \\
\hline & & $\mathrm{GL}$ & TA & $\mathrm{FL}$ & $\mathrm{GL}$ & TA & $\mathrm{FL}$ \\
\hline \multirow{2}{*}{$\begin{array}{l}\text { RMS } \\
\text { (pico) }\end{array}$} & pré & 100 & 100 & 100 & 100 & 100 & 100 \\
\hline & pós & $51(15 / 220)$ & $59(7 / 106)^{*}$ & $58(9 / 252)$ & $70(38 / 104)^{*}$ & $69(45 / 216)$ & $69(53 / 117)^{\star}$ \\
\hline \multirow{2}{*}{$\begin{array}{l}\text { RMS } \\
(0,2 \mathrm{~s})\end{array}$} & pré & $79(46 / 85)$ & $74(60 / 79)$ & $76(44 / 84)$ & $82(31 / 89)$ & $76(64 / 85)$ & $73(50 / 83)$ \\
\hline & pós & $41(13 / 116)$ & $43(5 / 71)^{*}$ & $49(6 / 205)$ & $57(30 / 81)^{*}$ & $53(34 / 151)$ & $47(37 / 84)^{*}$ \\
\hline \multirow{2}{*}{$\begin{array}{l}\text { RMS } \\
(1,0 \mathrm{~s})\end{array}$} & pré & $44(38 / 66)^{\#}$ & $42(38 / 58)^{\#}$ & $50(40 / 62)$ & $54(39 / 73)$ & $52(41 / 94)$ & $53(41 / 73)$ \\
\hline & pós & $28(11 / 131)$ & $24(3 / 116)^{\#}$ & $35(5 / 101)$ & $47(25 / 62)^{*}$ & $42(22 / 59)^{*}$ & $43(34 / 66)^{*}$ \\
\hline
\end{tabular}

*p<0,05 em relação ao respectivo músculo pré "p<0,05 em relação ao respectivo músculo do grupo não atleta; GL: gastrocnêmio lateral; TA: tibial anterior; FL: fibular longo; RMS: Root Mean Square 
indivíduos a tarefas que exigem componentes proprioceptivos, imediatamente após um tratamento de crioterapia. Também, Uchio et al. ${ }^{10}$ relatam a necessidade de preocupação com o retorno dos atletas aos exercícios após a crioterapia, considerando que os resultados são contraditórios, o que justifica a necessidade de estudos mais aprofundados.

Este estudo também teve por objetivo comparar os efeitos da crioimersão em atletas e não atletas. A hipótese inicial foi de que o treinamento esportivo de basquetebol, em função dos saltos, contatos físicos e atividades de mudanças rápidas de direção, forneceria aos atletas melhor controle motor e, dessa forma, a crioterapia teria menor efeito sobre o tornozelo, quando comparado aos indivíduos não atletas. Nesse sentido, Kirkendall e Garret Junior ${ }^{22}$ afirmam que um dos benefícios do treinamento esportivo, de acordo com a especificidade de cada modalidade esportiva, é a melhor sincronização da ativação das unidades motoras, que leva ao incremento do controle motor. Também, Cadore et al..$^{23}$ observaram aumento significativo na amplitude do sinal eletromiográfico após treinamento.

Entretanto, a hipótese não foi confirmada quando se analisou o pico do RMS e o RMS em até 0,2 segundos após a abertura da plataforma, pois evidenciou a igualdade dos grupos. Ao contrário, quando se analisou o RMS até 1 segundo após a abertura da plataforma, os atletas apresentaram menores valores de recrutamento para os músculos GL e TA, antes e após a aplicação da crioimersão. Justificando os resultados deste estudo, Bueno et al. ${ }^{24}$ afirmam que o treinamento específico de uma modalidade desportiva pode resultar em diferentes adaptações neuromusculares, de acordo com o estímulo empregado e assim, acreditamos que tais adaptações podem ser superiores ao déficit de controle motor causado pela crioimersão.

Existem algumas limitações que precisam ser reconhecidas e discutidas em relação ao presente estudo. Para a análise da resposta eletromiográfica após inversão do tornozelo a amostra foi composta por atletas e indivíduos ativos sem qualquer lesão no tornozdelo, pois os autores entendem que a plataforma de inversão poderia exacerbar a dor e/ou lesões em indivíduos com disfunções do tornozelo. Além disso, a possibilidade de um grupo controle submetido à imersão em água à temperatura ambiente ou em repouso deve ser considerada de forma a assegurar os efeitos da crioimersão na resposta neuromuscular do tornozelo.

\section{CONCLUSÃO}

De forma geral, o grupo de não atletas apresentou maiores alterações do sinal eletromiográfico após crioimersão, com menor recrutamento para os músculos GL, TA e FL; enquanto os atletas após o resfriamento estabeleceram menores valores somente para o TA. Como contribuição clínica, aponta-se que após o uso da crioterapia no tornozelo deve-se ter cuidado com o desenvolvimento de exercícios intensos, em função do menor recrutamento de unidades motoras.

\section{AGRADECIMENTOS}

Agradecemos ao Centro de Reabilitação do Hospital das Clínicas da Faculdade de Medicina de Ribeirão Preto, ao Conselho Nacional de Desenvolvimento Científico e Tecnológico (CNPq) - proc. 479616/20124 - e à Fundação de Amparo à Pesquisa do Estado de São Paulo (FAPESP) - proc. 10/09341-7.

\section{REFERÊNCIAS}

1. Marshall PWM, Mckee AD, Murphy BA. Impaired trunk and ankle stability in subjects with functional ankle instability. Med Sci Sports Exerc. 2008;41(8):1549-57.

2. Wilkerson GB, Horn-Kingery HM. Treatment of the inversion ankle sprain: comparison of different modes of compression and cryotherapy. J Orthop Sports Phys Ther. 1993;17(5):240-6.

3. Waterman BR, Belmont PJ Jr, Cameron KL, Deberardino TM, Owens BD. Epidemiology of ankle sprain at the United States Military Academy. Am J Sports Med. 2010;38(4):797-803.

4. Matheus JPC, Milani JGPO, Gomide LB, Volpon JB, Shimano AC. Análise biomecânica dos efeitos da croterapia no tratameto da lesão muscular aguda. Rev Bras Med Esporte. 2008;14(4):372-75.

5. Capps SG, Mayberry B. Cryotherapy and intermittent pneumatic compression for soft tissue trauma. Athl Ther Today. 2009;14(1):2-4

6. Enwemeka CS, Allen C, Avila P, Bina J, Konrade J, Munns S. Soft tissue thermodynamics before, during, and after cold pack therapy. Med Sci Sports Exerc. 2002;34(1):45-50

7. Santuzzi CH, Gonçalves WLS, Rocha SS, Castro MEC, Gouvea SA, Abreu GR. Efeitos da crioterapia, estimulação elétrica transcutânea e da sua associação na atividade elétrica do nervo femoral em ratos. Rev Bras Fisioter. 2008:12(6):441-46.

8. Oliveira R, Ribeiro F, Oliveira J. Cryotherapy impairs knee joint position sense. Int J Sports Med. 2010;31(3):198-201. 
9. Bleakley CM, O'Connor S, Tully MA, Rocke LG, Macauley DC; Mcdonough SM. The PRICE study (Protection Rest Ice Compression Elevation): design of a randomised controlled trial comparing standard versus cryokinetic ice applications in the management of acute ankle sprain. BMC Musculoskelet Disord. 2007:19(8):125.

10. Uchio Y, Ochi M, Fujihara A, Adachi N, Iwasa J, Sakai Y. Criotherapy influences joint laxity and position sense of the healthy knee joint. Arch Phys Med Rehabil. 2003;84(1):131-35.

11. Cordova ML, Bernard LWB, Au KK, Demchak TJ, Stone MB, Sefton $\mathrm{JM}$. Cryotherapy and ankle bracing effects on peroneus longus response during sudden inversion. J Electromyogr Kinesiol. 2010;20(2):348-53.

12. Berg CL, Hart JM, Palmieri-Smith R, Cross KM, Ingersoli CD. Cryotherapy does not affect peroneal reaction following sudden inversion. J Sport Rehabil. 2007;16(4):285-94.

13. Schmid S, Moffat M, Gutierrez GM. Effect of knee joint cooling on the electromyographic activity of lower extremity muscles during a plyometric exercise.. J Electromyogr Kinesiol. 2010;20(6):1075-81.

14. Surenkok O, Aytar A, Tuzun EH, Akman MN. Cryotherapy impairs knee joint position sense and balance. Iso and Exerc Sci. 2008:16(1):69-73.

15. Herrera E, Sandoval MC, Camargo DM; Salvini TF. Effect of walking and resting after three cryotherapy modalities on the recovery of sensory and motor nerve conduction velocity in healthy subjects. Rev Bras Fisioter. 2010;15(3):233-40.
16. Luzzati V, Mateu L, Marquez G, Borgo M. Structural and electrophysiological effects of local anesthetics and of low temperature onmyelinated nerves: implication of the lipid chains in nerve excitability. J Mol Biol. 1999;286(5):1389-402.

17. Herrera E, Sandoval MC, Camargo DM, Salvini TF. Motor and sensory nerve conduction are affected diferently by ice pack, ice massage, and cold water immersion. Phys Ther. 2010;90(4):581-591.

18. Khanmohammadi R, Someh M, Ghafarinejad F. The effect of cryotherapy on the normal ankle joint position sense. Asian J Sports Med. 2011;2(2):91-8.

19. Bleakley CM, Costello JT, Glasgow PD. Should athletes return to sport after applying ice? A systematic review of the effect of local cooling on functional performance. Sports Med. 2012;42(1):69-87.

20. Bahr R, Bahr IA. Incidence of acute volleyball injuries: a prospective cohort study of injuries mechanisms and risk factors. Scand J Med Sci Sports. 1991;7(3):166-71.

21. Costello JT, Donnelly AE. Cryotherapy and joint position sense in healthy participants: a systematic review. J Athl Train. 2010;45(3):306-316.

22. Kirkendall, DT, Garrett Junior, WE. A ciência do exercício e dos esportes. Porto Alegre: Ed. Artmed; 2003.

23. Cadore EL, Pinto RS, Lhullier FLR, Correa CS, Alberton CL, Pinto SS, et al. Physiological effects of concurrent training in elderly men. Int J Sports Med. 2010;31(10):689-97.

24. Bueno RC, Fortes JBP, Camacho SP. Eletromiografia do músculo quadríceps femural: influência do treinamento específico no disparo neuromotor periférico. Mov \& Percepção. 2007:11(8):55-70. 